\title{
Association mapping of quantitative trait loci for agronomic traits in spring wheat collection tested under two water regimes in Northern Kazakhstan
}

\author{
Amalova A. ${ }^{1,2 *}$, Abugalieva S. ${ }^{1,2}$, Babkenov A. ${ }^{3}$, Babkenova S. ${ }^{3}$, Turuspekov Y. ${ }^{1,2}$ \\ ${ }^{1}$ Institute of Plant Biology and Biotechnology, Almaty, Kazakhstan \\ ${ }^{2}$ Al-Farabi Kazakh National University, Almaty, Kazakhstan \\ ${ }^{3}$ A.I. Barayev Research and Production Centre for Grain Farming, Shortandy, Akmola region, \\ Kazakhstan \\ * email: akerke.amalova@gmail.com
}

Bread wheat is the most important cereal in Kazakhstan, where it grows on over 12 million hectares. One of the major constraints in the grain yield of wheat is a drought caused by a limited water supply. Hence, the development of drought-resistant cultivars will be critical for ensuring food security in this country. Therefore, identifying drought resistance-associated quantitative trait loci (QTLs) is an essential step in modern breeding activities that also rely on a marker-assisted selection approach. In this study, the collection of 179 spring wheat accessions were tested under irrigated and rainfed conditions in Northern Kazakhstan by using growth-related traits (heading date (HD), seed maturation date (SMD), plant height (PH), and etc.), and yield components, during three years (2018-2020). The collection was genotyped using a 20000 (20K) Illumina iSelect SNP array, and 8662 polymorphic SNP markers were selected for genome-wide association study (GWAS) to identify QTLs for targeted traits. Forty-six stable QTLs out of 237 total QTLs were identified for irrigated and rainfed conditions in the Akmola region, Northern Kazakhstan, by studying eight traits, including HD, SMD, PH, and grain yield components. Identified SNP markers of QTLs for studied traits can be directly utilized to develop new competitive spring wheat cultivars in Northern Kazakhstan. It was confirmed that GWAS is a powerful method in searching genes associated with the regulation of key agronomic traits in bread wheat.

Financial support: ИРН АР08855387 «Nested association mapping for gene discovery and deployment for improvement of yield, quality, and disease resistance in bread wheat» from the Ministry of Education and Science of the Republic of Kazakhstan. 